\title{
Estandarización de tasas de captura de pesquerías pelágicas de la zona norte de Chile durante 1987-92*
}

\author{
Eleuterio Yáñez R. ${ }^{1}$, Fernando Espíndola R. ${ }^{1}$, Pierre Fréon ${ }^{2}$, \\ María Angela Barbieri B. ${ }^{1,3}$ e Inés Guerrero S. ${ }^{4}$ \\ ${ }^{1}$ Escuela de Ciencias del Mar, Universidad Católica de Valparaíso \\ Casilla 1020, Valparaíso, Chile. E-mail: eyanez@ucv.cl \\ ${ }^{2}$ Institut de Recherches pour le Developpement, Francia \\ E-mail: pfreon@sfri.wcape.gov.za \\ ${ }^{3}$ Instituto de Fomento Pesquero. Huito 374, Valparaíso, Chile \\ E-mail: mabarbie@ifop.cl \\ ${ }^{4}$ Instituto de Estadística, Universidad Católica de Valparaíso \\ Blanco Viel 596, Valparaíso, Chile. E-mail: iguerrer@ucv.cl
}

Recibido 14 junio 1999; versión corregida 12 octubre 1999; aceptado 3 noviembre 1999.

RESUMEN. Se usó el modelo lineal general para estandarizar el índice de abundancia relativa, captura por unidad de esfuerzo (CPUE), de anchoveta (Engraulis ringens) y sardina (Sardinops sagax) en el norte de Chile $\left(18^{\circ} 21^{\prime}-24^{\circ} \mathrm{S}\right)$, entre 1987 y 1992. Para anchoveta se toma en cuenta la categoría de embarcación, la zona de pesca, la estación del año, el año y la temperatura superficial del mar (TSM). Para sardina se considera la categoría de embarcación, la zona de pesca, la estación del año y el año. Estas variables muestran en general efectos bastante significativos en el logaritmo de la CPUE. Además, interacciones significativas son identificadas para anchoveta entre año*estación, año*zona, estación*zona, zona*TSM, estación*año*zona, estación*año*TSM, estación*zona*TSM y año*zona*TSM. Para sardina las interacciones significativas identificadas son estación*año, estación*zona, año*zona y estación*año*zona. En conclusión, cerca del $35 \%$ y $45 \%$ de la variación total observada de la CPUE no fueron explicadas por los modelos planteados para ambas especies, debido probablemente al carácter aleatorio de la pesca.

Palabras claves: estandarización, CPUE, interacciones, anchoveta, sardina, norte de Chile.

\section{Standardization of catch rates for the northern Chile pelagic fishery during 1987-92}

\begin{abstract}
We used a general linear model for the standarization of the index of relative abundance catch per unit of effort (CPUE). The analysis was performed for anchovy (Engraulis ringens) and sardine (Sardinops sagax) in northern Chile (18 $\left.21^{\prime}-24^{\circ} \mathrm{S}\right)$, for the years 1987 through 1992. For anchovy we have considered ship hold capacity, fishing area, season, year and the sea surface temperature (SST). For sardine, we have considered ship's hold capacity, fishing area, season and year. These variables individually have a significant effect in the CPUE logarithms. For the anchovy, we identified a significant interaction between year*season, year*fishing area, season*fishing area, fishing area*SST, season*year*fishing area, season*year*SST, season*fishing area*SST, and year*fishing area*SST. In the case of the sardine the significant interactions identified were season*year, season*fishing area, and season*year*fishing area. In conclusion, approximately $35 \%$ to $45 \%$ of the total variation observed for the log of CPUE was not explained by the models. This is should be explained by the aleatory nature of the fishery.
\end{abstract}

Key words: standardization, CPUE, interactions, anchovy, sardine, Northern Chile.

\footnotetext{
* Proyecto FONDEF-EFISAT D98-I-1022.
} 


\section{INTRODUCCIÓN}

Los datos de captura por unidad de esfuerzo (CPUE) de la flota pesquera industrial de la zona norte de Chile son empleados frecuentemente para estimar índices de abundancia de anchoveta (Engraulis ringens) y sardina (Sardinops sagax) (Böhm et al., 1996). Estos índices son indicadores indirectos de la situación de los stocks, permitiendo una aproximación de la evolución de la abundancia de los recursos (Gascuel y Thiam, 1993); a la vez pueden ser utilizados como variables de entrada en los modelos de evaluación de stocks (Hilborn y Walters, 1992).

El uso de la CPUE como índice de abundancia ha sido muy criticado, dado el sesgo asociado a los cambios en el poder de pesca de las embarcaciones y a las variaciones espacio-temporales del patrón de explotación. Para minimizar este sesgo, normalmente se estiman factores potenciales de las diferentes categorías de embarcación, los que luego se emplean para estimar CPUE estandarizadas. Uno de los métodos más utilizados en el análisis de pesquerías es el modelo lineal (Gulland, 1956; Robson, 1966; Laurec y Fonteneau, 1979). Posteriormente se ha considerado el empleo del modelo lineal general (MLG), con el objeto de tomar en cuenta las interacciones siempre presentes entre las variables explicativas (Allen y Punsly, 1984; Punsly y Nakano, 1992; Gascuel y Thiam, 1993).

En el presente estudio se hace uso del MLG para estandarizar datos de CPUE de la pesquería de anchoveta y sardina de la zona norte de Chile, en el período 1987-92. Para tal efecto se estiman los poderes de pesca de las diferentes categorías de embarcación y luego los valores anuales del índice de abundancia relativa de dichos recursos.

\section{MATERIALES Y MÉTODOS}

La información pesquera analizada proviene de las bitácoras diarias de pesca disponibles de 33 embarcaciones, de 140 (6), 180 (1), 200 (2), 240 (4), 270 (1), $300(5), 330(10), 420$ (2) y 550 (2) $\mathrm{m}^{3}$ de capacidad de bodega. Dichas embarcaciones operaron normalmente en la zona norte de Chile $\left(18^{\circ} 21^{\prime}\right.$ $24^{\circ} \mathrm{S}$ ), entre 1987 y 1992 , logrando aproximadamente el $20 \%$ y $15 \%$ de las capturas totales de sardina y anchoveta respectivamente (Yáñez et al., 1993).

La información satelital disponible consistió en imágenes diarias de temperatura superficial del mar
(TSM), elaboradas con información captada a través del sensor AVHRR de satélites NOAA. Estas imágenes cubren el período 1987-1992 y la zona comprendida entre los $18^{\circ}$ y $23^{\circ} 30^{\prime} \mathrm{S}$, desde la costa hasta los $73^{\circ} \mathrm{W}$. Las imágenes representan matrices de datos de TSM de 256 líneas por 256 columnas, con una resolución de $2,2 \mathrm{~km}$ en el sentido nortesur y de 1,6 km en dirección este-oeste, en escala 1:2.000.000 (Barbieri et al., 1995). Para reducir el efecto de las nubes que se registran en dichas imágenes diarias, se emplea el valor máximo de la TSM (TSMM) de las cuadrículas con pesca (Pettigiani et al., 1992).

La forma de usar el MLG para estandarizar las tasas de captura es una modificación del método de Robson (1966), que consiste en usar los efectos de año del MLG directamente como estimación de abundancia (Punsly y Nakano, 1992). Para tal efecto se aplicó la transformación logarítmica a la variable CPUE, de tal forma que los residuos logren aproximadamente una distribución normal y permitan el uso de la prueba $F$ para determinar las variables significativas de los modelos analizados.

Dado que la distribución espacial del esfuerzo de pesca no es homogénea, para permitir el ajuste del modelo usando el efecto «barco» de las 33 embarcaciones, además del efecto no lineal de la variable continua capacidad de bodega, se considera la agrupación de barcos en categorías de acuerdo a la capacidad de bodega (Tabla 1). Por otra parte, dado que en ciertos meses las capturas son casi nulas debido a las vedas, se consideró que esta influencia externa se minimiza al agrupar trimestralmente la información. Además, de acuerdo con la información disponible, las zonas de pesca se asociaron a cuadrículas de 10x10 millas náuticas (Fig. 1).

Tabla 1. Categorías de embarcación según la capacidad de bodega $\left(\mathrm{m}^{3}\right)$.

Table 1. Categories of ships according to capacity of hold $\left(\mathbf{m}^{3}\right)$.

\begin{tabular}{|ccc|}
\hline Categoría & Anchoveta & Sardina \\
\hline 1 & $140-180$ & 140 \\
2 & $200-240-270$ & $180-200$ \\
3 & $300-330$ & $240-270-300$ \\
4 & 420 & 330 \\
5 & 550 & 420 \\
6 & & 550 \\
\hline
\end{tabular}

Fuente: Yáñez et al. (1993). 


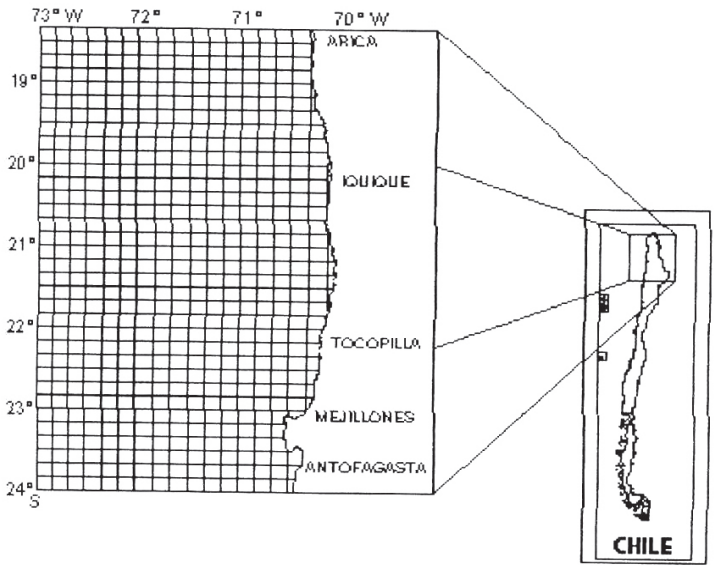

Figura 1. Zona de estudio dividida en cuadrículas de 10x10 millas náuticas.

Figure 1. Zone of study divided in $10 \times 10$ nautical miles grid.

Una vez establecidas las variables independientes (año, trimestre, categoría de embarcación, zonas de pesca y TSMM, se emplearon los software de análisis estadístico y matemático S-PLUS 4.5 (Mathsoft, 1998) y SPSS 8.0 para Windows (SPSS Inc., 1998), para llevar a cabo el procedimiento paso a paso de selección del modelo descrito por Allen y Punsly (1984). Este consiste en ajustar un modelo inicial con todas las variables e interacciones posibles, el cual se va simplificando al sacar aquellas variables e interacciones poco significativas.

\section{RESULTADOS Y DISCUSIÓN}

Del análisis se determinó que algunas variables e interacciones ejercen efectos significativos sobre la CPUE de anchoveta (Tabla 2). Para la sardina se encontraron resultados similares (Tabla 3), con la excepción del efecto de la temperatura que no fue significativo $(\mathrm{P}=0,68)$. De esta forma los modelos ajustados son, para anchoveta:

$$
\begin{gathered}
\mathrm{Y}_{i j k l}=\alpha+\mathrm{E}_{i}+\mathrm{A}_{j}+\mathrm{C}_{k}+\mathrm{D}_{l}+{ }_{\gamma} \mathrm{T}+\mathrm{EA}_{i j}+ \\
\mathrm{EC}_{i k}+\mathrm{AC}_{j k}+\mathrm{C}_{\mathrm{k} \gamma} \mathrm{T}+\mathrm{EAC}_{i j k}+ \\
\mathrm{EA}_{i j \gamma} \mathrm{T}+\mathrm{EC}_{i k \gamma} \mathrm{T}+\mathrm{AC}_{j k \gamma} \mathrm{T}+\in_{i j k l}
\end{gathered}
$$

$\mathrm{Y}_{i j k l}=$ logaritmo de la CPUE,

$\alpha \quad=$ promedio general,

$\mathrm{E}_{i} \quad=$ efecto del trimestre $i$,

$\mathrm{A}_{j} \quad=$ efecto del año $j$,

$\mathrm{C}_{k} \quad=$ efecto de la zona de pesca $k$,

$\mathrm{D}_{l}=$ efecto de la categoría de embarcación $l$,

$\gamma=$ coeficiente de regresión de la temperatura $\mathrm{T}$,

$\mathrm{EA}_{i j}=$ efecto de la interacción entre $\mathrm{E} i$ y $\mathrm{A} j$,

$\mathrm{EC}_{i k}=$ efecto de la interacción entre $\mathrm{E} i \mathrm{y} \mathrm{C} k$,

$\mathrm{AC}_{j k}=$ efecto de la interacción entre $\mathrm{A} j \mathrm{y} \mathrm{C} k$,

$\mathrm{C}_{k \gamma}=$ efecto de la interacción entre $\mathrm{C} k \mathrm{y} \mathrm{T}$,

$\mathrm{EAC}_{i j k}=$ efecto de la interacción entre $\mathrm{E} i, \mathrm{~A} j \mathrm{y} \mathrm{C} k$,

$\mathrm{EA}_{i j \gamma}=$ efecto de la interacción entre $\mathrm{E} i, \mathrm{~A} j \mathrm{y} \mathrm{T}$,

$\mathrm{EC}_{i k \gamma}=$ efecto de la interacción entre $\mathrm{E} i, \mathrm{C} k \mathrm{y} \mathrm{T}$,

$\mathrm{AC}_{j k \gamma}=$ efecto de la interacción entre $\mathrm{A} j, \mathrm{C} k \mathrm{y} \mathrm{T} \mathrm{y}$

$\epsilon_{i j k l}=$ error residual.

y para sardina:

$$
\begin{gathered}
\mathrm{Y}_{i j k l}=\alpha+\mathrm{E}_{i}+\mathrm{A}_{j}+\mathrm{C}_{k}+\mathrm{D}_{l}+\mathrm{EA}_{i j}+ \\
\mathrm{EC}_{i k}+\mathrm{AC}_{j k}+\mathrm{EAC}_{i j k}+\epsilon_{i j k l}
\end{gathered}
$$

$\mathrm{Y}_{i j k l} \quad=$ logaritmo de la CPUE,

$\alpha=$ promedio general,

$\mathrm{E}_{i} \quad=$ efecto del trimestre $i$,

$\mathrm{A}_{j} \quad=$ efecto del año $j$,

$\mathrm{C}_{k} \quad=$ efecto de la zona de pesca $k$,

$\mathrm{D}_{l}=$ efecto de la categoría de embarcación $l$,

$\mathrm{EA}_{i j}=$ efecto de la interacción entre $\mathrm{E} i$ y $\mathrm{Aj}$,

$\mathrm{EC}_{i k}=$ efecto de la interacción entre $\mathrm{E} i \mathrm{y} \mathrm{C} k$,

$\mathrm{AC}_{j k}=$ efecto de la interacción entre $\mathrm{A} j \mathrm{y} \mathrm{C}$,

$\mathrm{EAC}_{i j k}=$ efecto de la interacción entre $\mathrm{E} i, \mathrm{~A} j \mathrm{y} \mathrm{C} k$,

$\epsilon_{i j k l}=$ error residual.

Los modelos ajustados explican más de la mitad de la variación del logaritmo de la CPUE de anchoveta $\left(\mathrm{R}^{2}=0,65\right)$, y aproximadamente la mitad de la variación del logaritmo de la CPUE de sardina $\left(\mathrm{R}^{2}=0,55\right)$.

Las Figuras 2 y 3, a pesar de no mostrar una notoria homogeneidad de la varianza, tampoco indican un patrón sistemático con la transformación logarítmica, lo cual no invalida la prueba $F$. Los efectos principales mostrados en las Tablas 2 y 3 son en general altamente significativos, comparados con los efectos de las interacciones de primer y segundo orden. No obstante, el efecto de las interacciones presentes en los modelos ajustados toma en cuenta el $47 \%$ y $51 \%$ de la variación total explicada por los 


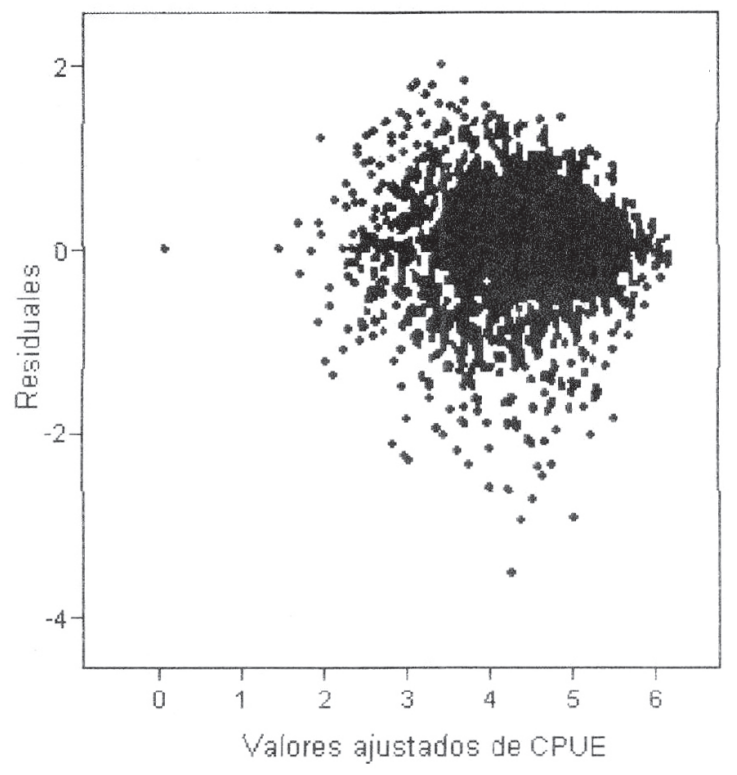

Figura 2. Residuales en función de las CPUE ajustadas de anchoveta según el modelo ajustado (1).

Figure 2. Residuals versus predicted values of CPUE for anchovy according to fitted model (1).

modelos seleccionados para anchoveta y sardina, respectivamente. Cabe señalar que para ambos recursos no se observaron interacciones significativas involucrando la variable categoría de embarcación. No obstante, esta variable en forma individual es altamente significativa, particularmente en sardina (Tabla 3).

Por otra parte, cabe indicar que la incorporación de los efectos del año en las interacciones de los modelos seleccionados impide el logro de estimaciones insesgadas de las variaciones anuales de la CPUE. En este caso se deben descartar estas interacciones de los modelos, considerando sus efectos como un residuo en un modelo sub-óptimo.

Así, durante el período de estudio se distingue para anchoveta el bajo nivel del índice de abundancia en 1987 (Fig. 4), lo cual se asocia al importante evento "El Niño" acontecido durante este año (Yáñez et al., 1995). El índice de anchoveta aumenta notablemente en 1988-89, asociado a años más normales desde el punto de vista de la estructura térmica superficial. Posteriormente este índice vuelve a disminuir, al mismo tiempo que se produce un notable enfriamiento en 1990 y fenómeno "El Niño" de regular intensidad en 1991-92, responsable en alguna medida de la mayor dispersión del índice de abundancia en 1992.

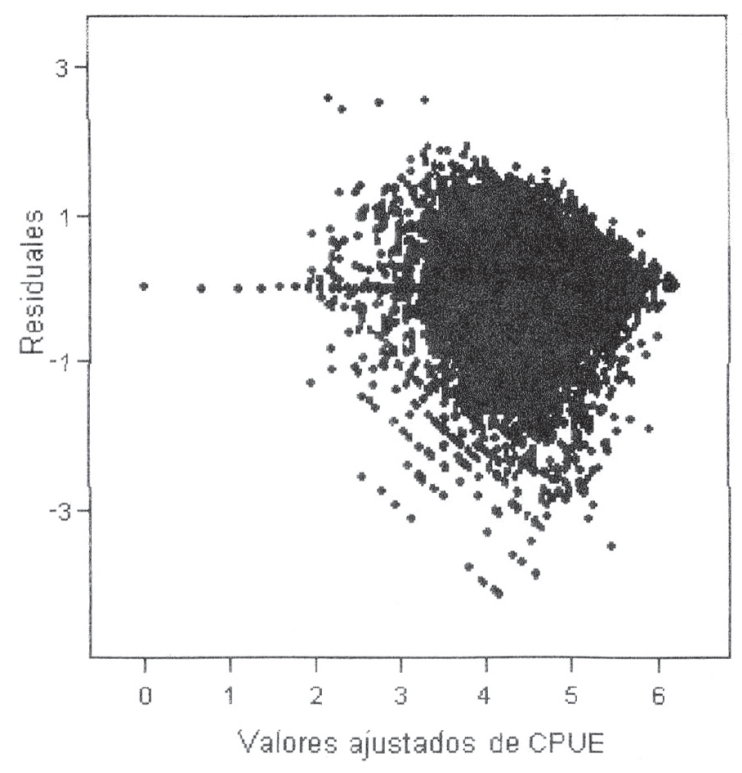

Figura 3. Residuales en función de las CPUE ajustadas de sardina según el modelo ajustado (2).

Figure 3. Residuals versus predicted values of CPUE for sardine according to fitted model (2).

Los primeros años del período de estudio muestran los mayores valores del índice de abundancia de sardina, en tanto que después de 1989 el índice disminuye notablemente (Fig. 5). Esto concuerda con la evolución de los desembarques, los cuales pasan de un promedio de 1.576.000 $\mathrm{t}$ en 1987-89, a 694.000 t en 1990-92 (SERNAP, 1987-92).

Las diferentes categorías de embarcación consideradas no presentan una relación directa con el efecto individual que ejercen sobre el logaritmo de la CPUE de ambas especies (Fig. 6 y 7). Para anchoveta la categoría dos presenta una mayor eficiencia de pesca global, particularmente con respecto a la categoría uno considerada como estándar. Para sardina, las categorías tres y cuatro presentan la mayor eficiencia de pesca global, sobre todo en relación con la categoría uno.

Normalmente se espera que los poderes de pesca presenten una relación directa con el tamaño de la capacidad de bodega de las embarcaciones, debido a la eliminación progresiva del efecto de saturación (referido a la combinación arte-bodega). En este sentido, las Figuras 6 y 7 sugieren que esta eliminación desaparece a partir de la categoría 2 , indicando que los barcos de mayor tamaño no serían más eficientes. Esto puede deberse a problemas en la relación arte-bodega y a una menor "habilidad" de los 
Tabla 2. Análisis de varianza del logaritmo de la CPUE de anchoveta, según el modelo ajustado para el período 1987-92 (1).

Table 2. Analysis of variance of logarithms of the CPUE from anchovy, according to fitted model for period 1987-92 (1).

\begin{tabular}{|c|ccccc|}
\hline & $\begin{array}{c}\text { Grados de } \\
\text { Libertad }\end{array}$ & $\begin{array}{c}\text { Suma de } \\
\text { Cuadrados }\end{array}$ & $\begin{array}{c}\text { Cuadrado } \\
\text { Medio }\end{array}$ & Valor F & Probabilidad \\
\hline Trimestre(E) & 3 & 114,126 & 38,042 & 69,57 & 0,000 \\
Año(A) & 5 & 219,687 & 43,937 & 80,35 & 0,000 \\
Cuadrícula(C) & 115 & 336,877 & 2,929 & 5,36 & 0,000 \\
Categoría(D) & 4 & 152,742 & 38,185 & 69,83 & 0,000 \\
TSMM(T) & 1 & 34,358 & 34,358 & 62,83 & 0,000 \\
E x A & 13 & 52,441 & 4,034 & 7,38 & 0,000 \\
E x C & 110 & 235,993 & 2,145 & 3,92 & 0,000 \\
A x C & 83 & 138,742 & 1,672 & 3,06 & 0,000 \\
C x T & 46 & 95,209 & 2,069 & 3,79 & 0,000 \\
E x A x C & 20 & 40,841 & 2,042 & 3,73 & 0,000 \\
E x A x T & 20 & 85,406 & 4,270 & 7,81 & 0,000 \\
E x C x T & 21 & 60,524 & 2,882 & 5,27 & 0,000 \\
A x C x T & 13 & 45,513 & 3,501 & 6,40 & 0,000 \\
Residual & 1605 & 877,647 & 0,5468 & & \\
\hline
\end{tabular}

Tabla 3. Análisis de varianza del logaritmo de la CPUE de sardina, según el modelo ajustado para el período 1987-92 (2).

Table 3. Analysis of variance of logarithms of the CPUE from sardine, according to fitted model for period 1987-92 (2).

\begin{tabular}{|c|ccccc|}
\hline & $\begin{array}{c}\text { Grados de } \\
\text { Libertad }\end{array}$ & $\begin{array}{c}\text { Suma de } \\
\text { Cuadrados }\end{array}$ & $\begin{array}{c}\text { Cuadrado } \\
\text { Medio }\end{array}$ & Valor F & Probabilidad \\
\hline Trimestre(E) & 3 & 3,200 & 1,067 & 11,10 & 0,000 \\
Año(A) & 5 & 9,080 & 1,816 & 18,91 & 0,000 \\
Cuadrícula(C) & 407 & 170,622 & 0,419 & 4,36 & 0,000 \\
Categoría(D) & 5 & 147,741 & 29,548 & 307,62 & 0,000 \\
E x A & 15 & 9,420 & 0,628 & 6,54 & 0,000 \\
E x C & 356 & 104,994 & 0,295 & 3,07 & 0,000 \\
A x C & 519 & 186,199 & 0,359 & 3,74 & 0,000 \\
Ex A x C & 129 & 44,085 & 0,342 & 3,56 & 0,000 \\
Residual & 8375 & 804,448 & 0,0961 & & \\
\hline
\end{tabular}

barcos de mayor tamaño, producto de su propio tamaño y de entradas más recientes a la pesquería, lo cual explicaría la mayor dispersión de la última categoría compuestas sólo de dos embarcaciones.

Los mayores rendimientos se obtuvieron en cuadrículas de pesca con TSMM de $22-24^{\circ} \mathrm{C}$ y no en cuadrículas con TSMM de $17-20^{\circ} \mathrm{C}$ donde se concentra la mayor actividad de pesca de anchoveta (Fig. 8). La relación lineal que existe entre el logaritmo de la CPUE y la TSMM muestra un in- cremento de 0,053 unidades por unidad de aumento de la TSMM, sobre la media general; adicional al efecto que ejerce la TSMM al interactuar con otras variables. Es decir, los cambios en la temperatura superficial del mar tienen un efecto significativo en las capturas de anchoveta (Tabla 2).

Los cambios de la temperatura superficial del mar se asociarían con las variaciones de la distribución y abundancia de los recursos. Durante los años más bien fríos los recursos presentan una distribu- 


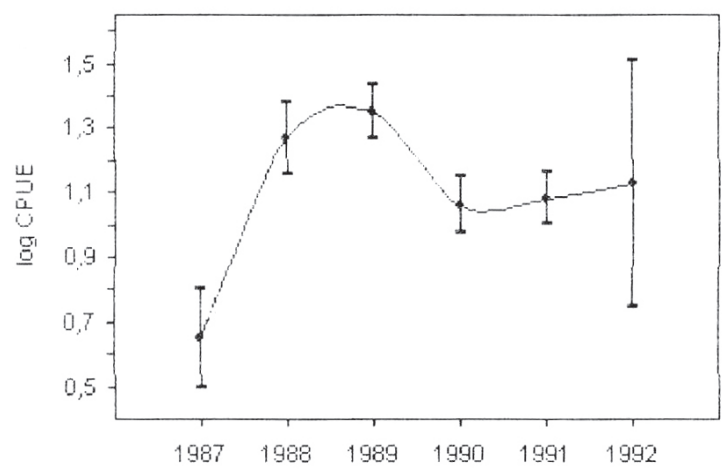

Figura 4. Variación anual del índice de abundancia de anchoveta, con intervalos de confianza al $95 \%$.

Figure 4. Annual variations of the index abundance for anchovy with $95 \%$ confidence interval.

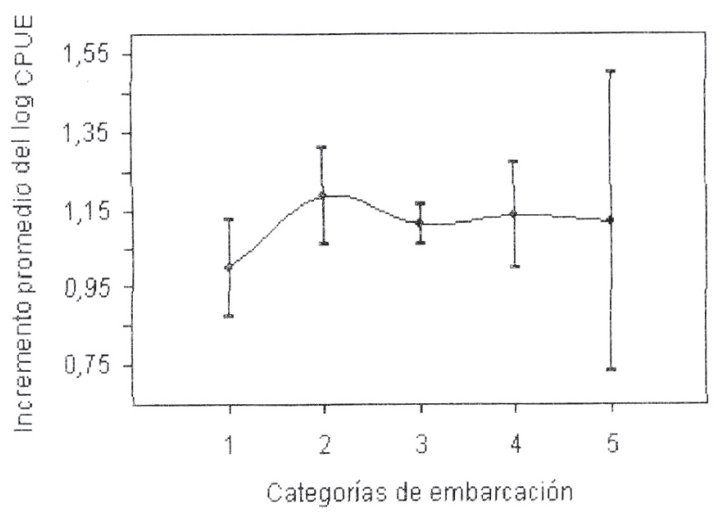

Figura 6. Poderes de pesca de embarcaciones que capturan anchoveta, con intervalos de confianza al $95 \%$. Figure 6. Fishing power for the ship's category referred to anchovy, with $95 \%$ confidence interval.

ción más amplia, en tanto que durante los eventos "El Niño" éstos se concentran en la costa aumentando la vulnerabilidad y por ende el rendimiento de las embarcaciones. Esto puede explicar las interacciones entre la temperatura y las cuadrículas donde se realizan faenas de pesca.

El efecto estacional del logaritmo de la CPUE varía entre años (Fig. 9 y 10). Al comienzo del período de estudio el logaritmo de la CPUE de sardina presenta los mayores valores en el segundo trimestre (abr-jun) y los menores en el cuarto (oct-dic). Al final del período, los mayores valores se registran en el primer trimestre (ene-mar) y los menores en el tercero (jul-sep). En cambio, para anchoveta se observa un patrón de abundancia estacional con los

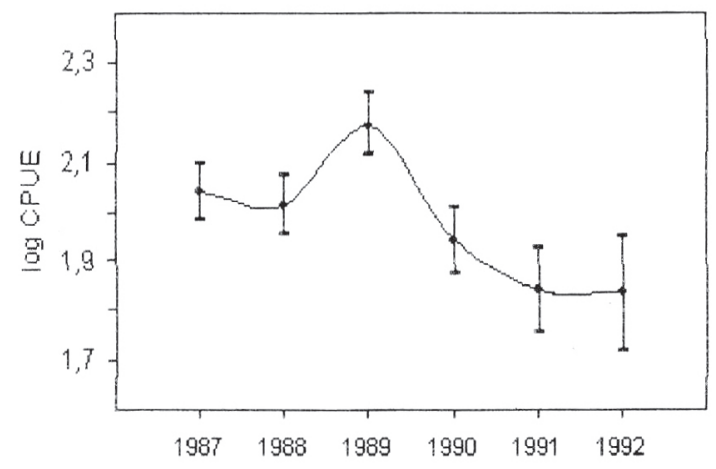

Figura 5. Variación anual del índice de abundancia de sardina, con intervalos de confianza al $95 \%$.

Figure 5. Annual variations of the index abundance for sardine with the $95 \%$ confidence interval.

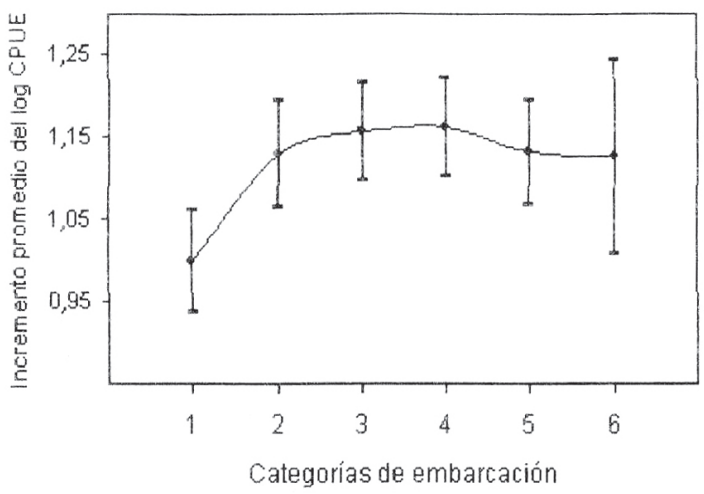

Figura 7. Poderes de pesca de embarcaciones que capturan sardina, con intervalos de confianza al $95 \%$.

Figure 7. Fishing power for the ship's category referred to sardine with $95 \%$ confidence interval.

valores más altos en el primer trimestre (ene-mar) y los más bajos en el cuarto (oct-dic), con excepción del año más bien frío de 1990.

Los recursos pelágicos analizados presentan variaciones espaciales intra e interanuales, las cuales se relacionan con procesos biológicos y fluctuaciones del medio ambiente (Yáñez et al., 1995). En efecto, los eventos de surgencia, la variabilidad de la estructura térmica y el aperiódico fenómeno "El Niño", son factores que condicionan la distribución y abundancia de estos recursos.

La interacción entre los efectos año y cuadrícula presentes en los modelos planteados indican que la distribución espacial de la densidad de anchoveta y sardina no permanece constante de año en año (Fig. 


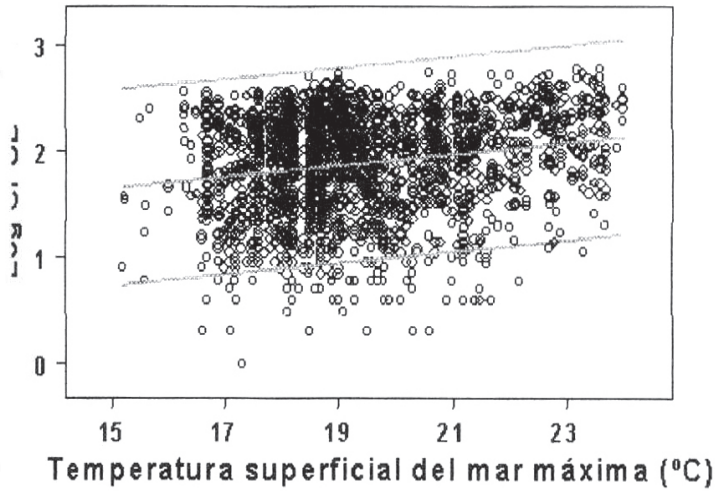

Figura 8. Relación entre el logaritmo de la CPUE de anchoveta y la TSMM de la cuadrícula correspondiente, con intervalos al $95 \%$ de confianza.

Figure 8. Relationship between the logarithms of the anchovy CPUE and sea surface temperature maximum of the corresponding grid, with $95 \%$ confidence interval.

11 y 12), lo cual coincide con lo mostrado por Yáñez et al. (1993, 1995). Del mismo modo, la interacción entre los efectos año y trimestre implica que el patrón estacional de densidad de sardina y anchoveta tampoco es constante en el período 1987-92 (Fig. 9 y 10). Este comportamiento estacional sería causado por la fuerte agregación con fines reproductivos que presentan ambos recursos y por los cambios del ambiente asociados a la ocurrencia de eventos fríos y cálidos.

Los modelos desarrollados en el presente estudio parecen adecuados para estimar índices de abundancia basados en la CPUE, no obstante que es recomendable el uso de una mayor cantidad de embarcaciones de la flota cerquera de la zona norte de Chile. No obstante, otros análisis de datos con MLG muestran que la proporción de la variación de la CPUE no explicada por el modelo es bastante considerable: $90 \%$ para el atún aleta amarilla capturado con cerco en el Pacífico oriental (Allen y Punsly, 1984); $45 \%$ para el atún patudo y $67 \%$ para el atún aleta amarilla capturado con palangre (Punsly y Nakano, 1992); y 60\% para lenguado (Large, 1992). Lo mismo ocurre con el atún aleta azul (Sainsbury et al., 1997), y los stocks de morue, merlan, eglefin y lieu noir (Millischer y Gascuel, 1997).

Cabe recordar que en el presente estudio el 35\% y $45 \%$ de la variación total observada en el logaritmo de la CPUE no fueron explicados por los modelos ajustados para anchoveta y sardina respectivamente. Estos porcentajes podrían deberse, entre otros

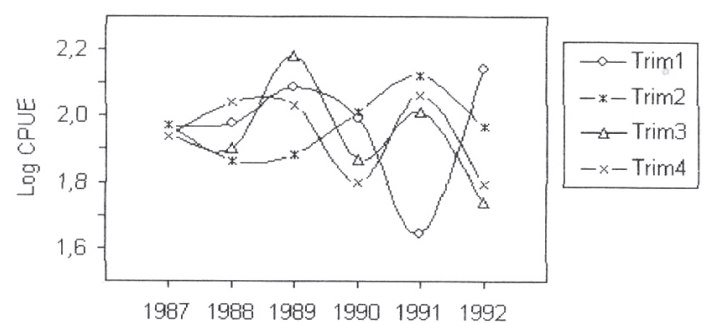

Figura 9. Interacción entre trimestre y año para sardina.

Figure 9. Interaction between the season and year for sardine.

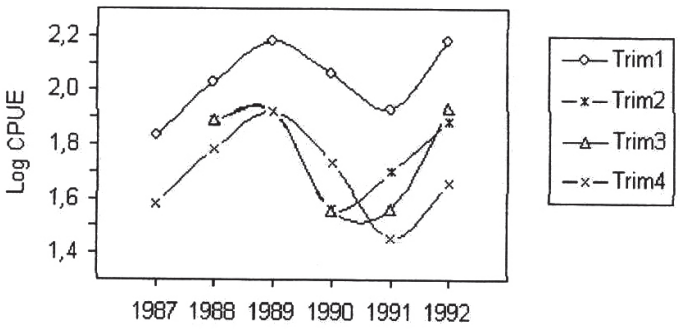

Figura 10. Interacción entre trimestre y año para anchoveta.

Figure 10. Interactions between the season and year for anchovy.

motivos, a la gran variabilidad en la probabilidad de encuentro entre los barcos y los cardúmenes, y la variabilidad en el tamaño que presentarían los cardúmenes. Sin embargo, explicar un $65 \%$ de la variabilidad de un conjunto de 2.050 observaciones de CPUE de anchoveta y un 55\% de 9.960 observaciones de CPUE en sardina, resultan porcentajes bastante apreciables.

De cualquier forma, una mayor ventaja del experimento proyectado debiera basarse en el análisis de matrices de datos más equilibradas, sin limitación sobre los cálculos de las interacciones y por ende con mejor precisión en los resultados. El desbalance que presentan los datos analizados se debería en gran medida a la extensa zona de estudio y al número de barcos considerados (33), los cuales 


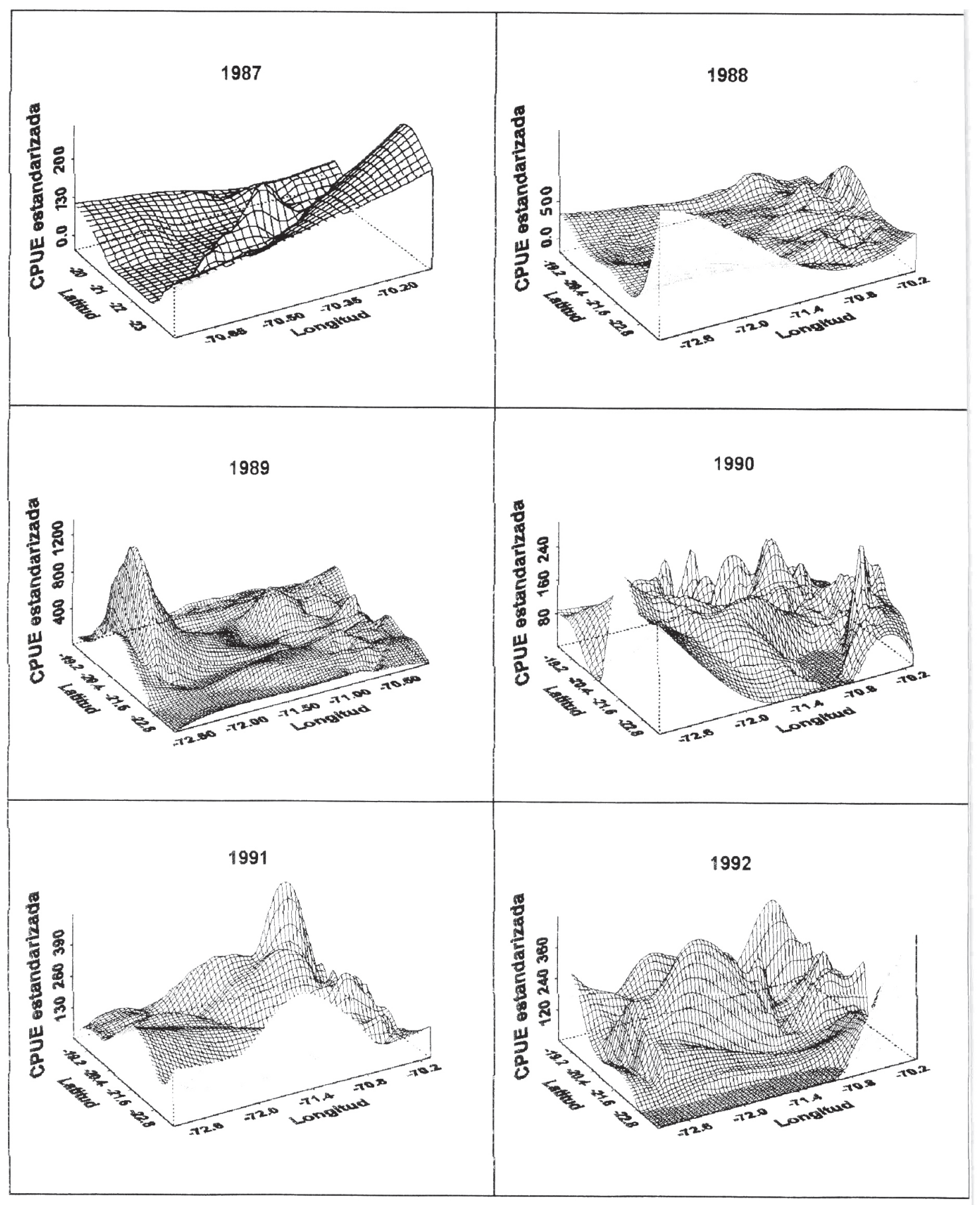

Figura 11. Plano de superficie de la CPUE estandarizada para anchoveta.

Figure 11. Surface's plane of CPUE standarized for anchovy. 


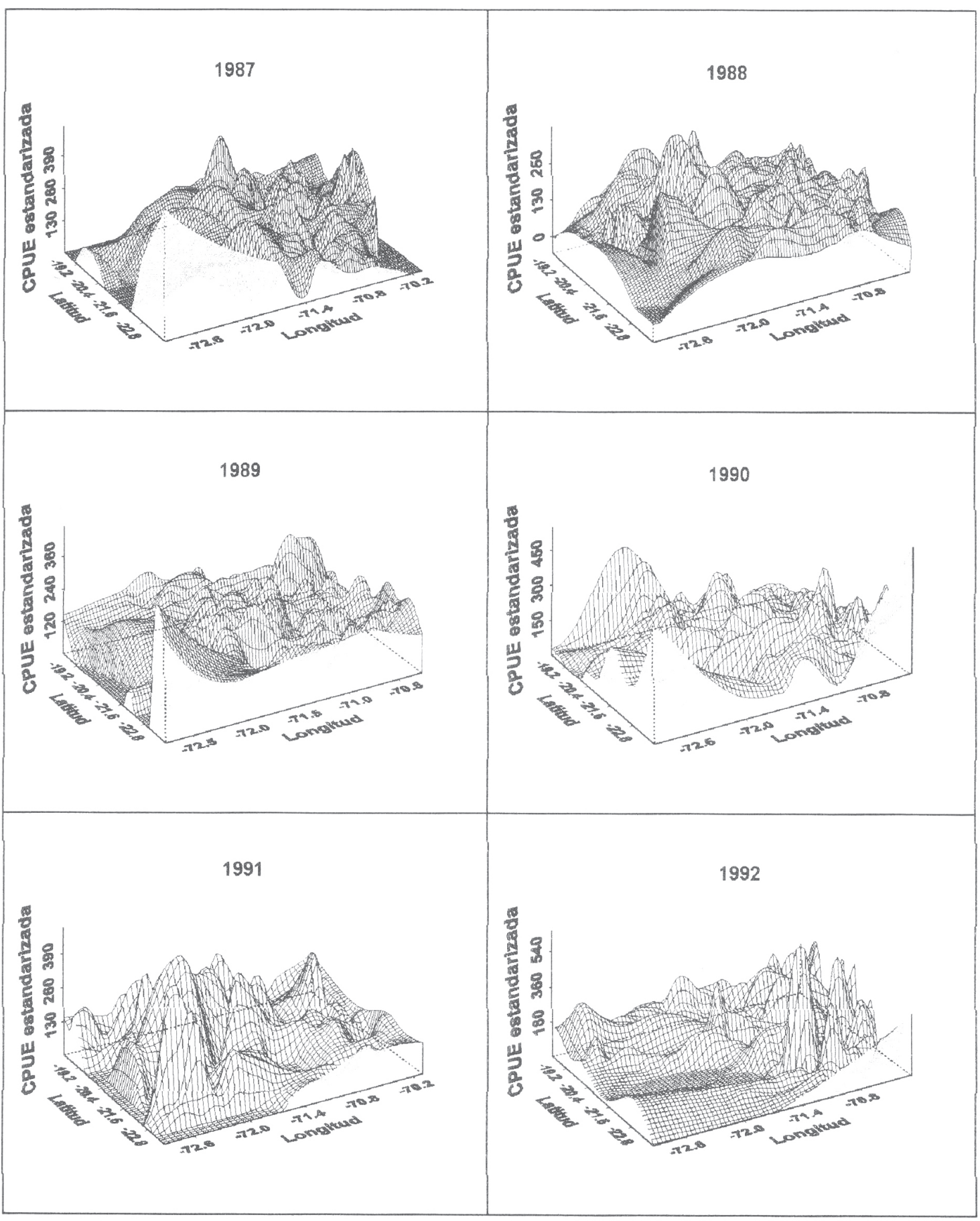

Figura 12. Plano de superficie de la CPUE estandarizada para sardina.

Figure 12. Surface's plane of CPUE standarized for sardine. 
no habrían sido capaces de visitar todas las cuadrículas y en todos los trimestres del período considerado. El porcentaje relativamente bajo de la varianza explicada se debería al carácter aleatorio de la pesca, sobre todo en este caso que trata sobre capturas de recursos pelágicos. Finalmente, se debe señalar que durante eventos "El Niño" se producen notables disminuciones de la abundancia en la zona de estudio, particularmente de anchoveta.

De acuerdo con Gauthiez (1995), la utilización del MLG presenta el problema de no estar basado en el análisis de procesos explicativos. Es difícil en este tipo de análisis incorporar un modelo del conocimiento o de las hipótesis particulares sobre el comportamiento del recurso, o sobre los procesos de captura.

No obstante, es recomendable el uso de este tipo de modelos en el análisis de datos de CPUE de éstas y otras pesquerías chilenas. En forma más particular, un factor sobre el tipo de equipo de pesca empleado (Americano, Abas, Petrel, etc.) puede ser incluido, de manera de no restringir el análisis a uno sólo de carácter general. De esta manera se podrían integrar los cambios que ocurren en una pesquería por el avance tecnológico a través de los años, considerando las características más relevantes (tamaño de redes, equipos acústico, GPS, etc.), el aumento de la habilidad de la tripulación, u otras características de las embarcaciones que podrían incluirse en la modelización.

\section{REFERENCIAS}

Allen, R.L. y R.G. Punsly. 1984. Catch rates as indices of abundance of yellowfin tuna, Thunnus albacares, in the eastern Pacific Ocean. Inter-Amer. Trop. Tuna Comm., Bull., 18(4): 301-379.

Barbieri, M.A., M. Bravo, M. Frías, A. González, O. Pizarro y E. Yáñez. 1995. Fenómenos asociados a la estructura térmica superficial del mar observados a través de imágenes satelitales en la zona norte de Chile. Invest. Mar., Valparaíso, 23: 99122.

Böhm, G., M.A. Barbieri, E. Yáñez, A. González, C. Canales y V. Catasti. 1996. Análisis de la captura y del esfuerzo de pesca de las unidades de pesquería de sardina española y anchoveta de la zona norte. Informe Final Proyecto FIP N ${ }^{\circ} 24 / 94$, IFOP-UCV, 111 pp. y Anexos.
Gascuel, D. y M. Thiam. 1993. Evolution de 1'abondance des ressources démersales sénégalaises: estimation par modélisation linéaire des PUE. In: M. Barry-Gérard, T. Diouf et A. Fonteneau (eds.). L'evaluation des ressources exploitables par la pêche artisanale sénégalaise. Tomo 2. Symposium de Dakar, du 8 a 13 février 1993.pp. 191-213.

Gauthiez, F. 1995. Estimation des puissances de pêche. In: J. Ferraris, D. Pelletier, Marie-Joelle Rochat (eds.). Méthodes D’etude des systémes halieutiques et aquacoles. Actes du Deuxiéme Forum Halieumétrique, Nantes du 26 au 28 juin 1995.

Gulland, J. 1956. On the fishing effort in English demersal fisheries. Fish. Invest., MAFF London, Series 2, 20(5): 1-41.

Hilborn, R. y C.J. Walters. 1992. Quantitative fisheries stock assessment: Choice, dynamics and uncertainty. Chapman and Hall. New York, 570 pp.

Large, P.A. 1992. Use of a multiplicative model to estimate abundance from commercial CPUE data. ICES J. mar. Sci., 49: 253 - 261.

Laurec, A. y A. Fonteneau. 1979. Estimation de l'abondance d'une clase d'âge: utilisation des C.P.U.E. de plusieurs engins, en differentes zones et saisons. "Collective Volume of Scientific Papers", International Commission for the Conservation of Atlantic Tunas, Vol. VIII, N ${ }^{\circ} 45$, 117 pp.

Laurec, A. y J.C. Le Guen. 1981. Méthodes de base en dynamique des populations marines exploitées. Tome I. Concepts fondamentaux, modeles essentiels, principes d'échantillonnage. Seminaire de Dynamique de Populations. G.S.G., Brest (Francia), 8-26 Septembre 1980.

Mathsoft, 1998. Software de análisis estadístico y matemático S-PLUS 4.5.

Millischer, L. y D. Gascuel. 1997. Estimation des puissances de pêche: Etude des flotilles industrielles de Bretagne Sud. In: LA SUREXPLOITATION. Troisiéme Forum de L'Association Francaise d'Halieumétrie. Montpellier, 1-3 juillet 1997.

Pettigiani, E., H. Karszenbaum y M. Mejail. 1992. Análisis de imágenes satelitarias de temperaturas superficiales mediante funciones empíricas ortogonales en la zona común de pesca argentinouruguaya. Revista SELPER 8(1): 43-48. 
Punsly, R. y H. Nakano. 1992. Analysis of variance and standarization of longline hook rates of bigeye (Thunnus obesus) and yellowfin (Thunnus albacares) tunas in the eastern pacific ocean during 1975-1987. Inter-Amer. Trop. Tuna Comm., Bull., 20(4): 179-184.

Robson, D.S. 1966. Estimation of the relative fishing power of individual ships. ICNAF, Res. Bull., 3, 5 -14 .

Sainsbury, K., T. Polacheck, N. Klaer, J. Gunn, R. Campbell, W. Hearn, T. Davis, A. Betlehem, A. Preece y A. Cowling. 1997. Recent developments and methods in the southern bluefin tuna fishery assessments. CSIRO, Division of Marine Research, Hobart, Australia.
SERNAP. 1987-92. Anuarios estadísticos de pesca. Servicio Nacional de Pesca, Ministerio de Economía, Chile.

SPSS Inc. 1998. SPSS 8.0 para Windows. Estadísticas Reales, Realmente Sencillas.

Yáñez, E., C. Canales, M.A. Barbieri, A. González y V. Catasti, 1993. Estandarización del esfuerzo de pesca y distribución espacial e interanual de la CPUE de anchoveta y sardina en la zona norte de Chile 1987 y 1992. Invest. Mar., Valparaíso, 21:111-132.

Yáñez, E., A. González y M.A. Barbieri. 1995. Estructura térmica superficial del mar asociada a la distribución espacio-temporal de sardina y anchoveta en la zona norte de Chile entre 1987 y 1992. Invest. Mar., Valparaíso, 23: 123-147. 